\title{
Toxic Plants: Knowledge, Medicinal Uses and Potential Human Health Risks
}

\author{
Rita Serrano \\ Research Institute for Medicines (iMed.ULisboa), Faculty of Pharmacy, Universidade de Lisboa, Portugal
}

Copyright $\mathrm{C} 2018$ by authors, all rights reserved. Authors agree that this article remains permanently open access under the terms of the Creative Commons Attribution License 4.0 International License

\begin{abstract}
A toxic plant is a plant that when touched or ingested in sufficient quantity can be harmful or fatal to man or other animals. When used in small amounts and correct proportions, these plants can be used as herbal drugs with medicinal properties. According to official sources from several countries, injurious and poisonous plants are responsible for many cases of intoxications worldwide. The beauty of these plants hides their toxicity and the unawareness potentiates these situations. Therefore, this bibliographic revision is about examples of some plants associated with poisoning but used for their medicinal properties, focusing on species with anticholinergic poisons (Atropa belladonna L., Datura stramonium L., Hyoscyamus niger L.), with calcium oxalate crystals (Philodendron spp.), with cardiac glycosides (Digitalis purpurea L.), with convulsant poisons and with nicotine-like alkaloids (Conium maculatum L., Nicotiana glauca Graham), with mitotic inhibitors (Catharanthus roseus L., Colchicum autumnale L.), with pyrrolizidine alkaloids (Senecio vulgaris L.), with sodium channel activators (Aconitum napellus L., Rhododendron spp.), with cyanogenic compounds (Hydrangea macrophylla (Thunb.) Ser.), and with toxalbumins (Abrus precatorius L., Ricinus communis L.). The knowledge about toxic plants is still insufficient and makes it so important to characterize some species that cause intoxication and lethality in humans.
\end{abstract}

Keywords Herbal Medicine, Poisonous Plants, Toxic Mechanisms, Toxic Plants, Toxicity

\section{Introduction}

Toxic plants are plants that have in their constitution chemical compounds or active principles, which through contact, inhalation or ingestion, are capable of causing injury, disease and even death in humans and animals. These compounds may be alkaloids, glycosides, saponins, oxalates, tannins, among others. [1] These compounds are part of the secondary metabolites of plants and although they are considered toxic to humans and certain animals, they have a reason to exist because they play important roles in protecting the plant against certain predators and pathogens.

The toxicity of plants differs from plant to plant and depends on several factors, namely the different chemicals that characterize it. The part of the plant ingested, its concentration, the chemical substances present, the age, the physical conditions for its development (type of soil, humidity, temperature, time of year), and the ripening state of its fruits are aspects to consider. [1].

In case of intoxication it is important to take into account our own interpersonal variability, where characteristics such as age, weight, physical condition and degree of exposure to the plant are fundamental and sometimes determinant.

Fortunately, most poisonings are characterized by irritations of the gastrointestinal tract, such as nausea, vomiting, diarrhea and some dermatological discomfort, such as dermatitis. There are, however, more severe poisonings in which cytotoxicity or central nervous system depression can occur, which can lead to respiratory and cardiac failure, leading to death.

The best way to minimize accidental intoxication with toxic plants is to be aware of them. This knowledge should be disseminated by the population in general, especially to childhood educators and to official entities that do mapping drawings of parks and gardens. [2].

In case of accidental ingestion, the remainder of the plant should be removed from the mouth, which should be rinsed with water. The plant must be stored for identification so that the most appropriate treatment can be obtained. It is important to avoid inducing vomiting, as it may cause blockage of the glottis and suffocation. [2]

This study aims to present a general review of some plants associated with poisoning but used for their medicinal properties.

\section{Materials and Methods}

Relevant literature was reviewed by probing scientific 
databases (PubMed, Scopus, Google Scholar, b-on, Web of knowledge) and books. The focus is the characterization of plants that cause potential human health risks. Some example plants were selected and organized based on their chemical properties and substances, together with context about their harmful effects and possible medicinal applications, historical and geographical context, relevant facts and curiosities, and typical intoxication cases and dosages.

\section{Results}

\subsection{Species with Anticholinergic Poisons}

These agents have a competitive antagonism of acetylcholine at the muscarinic subtype of the acetylcholine receptor, which is primarily located in the parasympathetic nervous system and the brain. [3]

Atropa belladonna L., also known as belladonna or devil herb, belongs to the botanical family of Solanaceae. It is an autochthonous plant in Portugal and can also be found in Europe, North Africa and West Asia. [4,5]

Belladonna has anticholinergic toxicity. This toxicity is due to the presence of three tropic alkaloids, these being atropine, scopolamine and hyoscyamine. These alkaloids can be found throughout the plant, i.e., in roots, leaves and berries. Berries are more relevant because they are easily ingested, especially by children and animals. [5]

The symptoms of this intoxication may be caused by both central nervous system and peripheral nervous system effects.

The effects at the central level are dose dependent and include ataxia, disorientation, agitation, memory loss, visual and auditory hallucinations, delusions, convulsions, coma, and ultimately heart and respiratory failure. Peripheral effects include mydriasis, red skin, dry mucous membranes, urinary retention, tachycardia, and others. [5]

The treatment of intoxication is essentially symptomatic. For example, antiemetics and activated charcoal may be used, and it is also important to provide oxygen and electrolytes. In more severe cases, physostigmine can be administered. [5,6]

Due to its hallucinogenic effects, this plant is also used for recreational purposes. In Europe, during the Middle Ages, this plant was widely used for the production of ointments for the practice of witchcraft, and it is also associated with prophecies and divinations due to the fact that hallucinations can be understood and confused with reality. [7]

There are cases of accidental intoxication by both children and adults as well as cases of suicide.

Datura stramonium L. belongs to the family Solanaceae, is commonly known as fig-hell, dwarf, devil herb, prickly pear, among others. It is a species of exotic naturalness, but one that can easily be found in Portugal, is distributed across all regions of the country and is considered an invasive species. [4]

This toxic plant causes anticholinergic syndrome, being hallucinogenic due to the presence of toxic alkaloids, namely atropine, hyoscyamine and scopolamine. The whole plant is toxic, especially its seeds. [8]

The most common symptoms are auditory and visual hallucinations, disorientation, strained voice, agitation, tachycardia, fever, dry skin, dilated pupils. There are, however, more severe cases in which seizures and urinary retention can occur. [9]

It is important to mention that visual hallucinations usually indicate cases of toxicity, while hearing disorders may also indicate another type of pathology such as schizophrenia, however, the visual hallucinations do not always manifest themselves in this type of intoxication, so they should be considered with caution for the correct diagnosis of intoxication by this plant. [9].

Symptoms of intoxication by this plant may begin to appear sixty minutes after ingestion and may be prolonged for the next 24-48 hours due to delayed gastric emptying. [10]

Its use may be conscious, being widely used among adolescents due to its hallucinogenic effects, however accidental poisoning also occurs in children due to ingestion of the plant. [9]

Hyoscyamus niger L. belongs to the Solanaceae family and is commonly known as black meimendro. It is a species native to Europe and North Africa, being native to Portugal. It can be found in sea cliffs, embankments, walls, and also in pastures. $[8,11]$.

Its toxicity is also due to the presence of the tropic alkaloids, i.e., hyoscyamine, atropine and especially scopolamine, and the whole plant is toxic. Its symptoms and treatment are similar to those already described. [11]

There are cases of accidental intoxication, especially in children, and intentional in cases of suicide or due to its hallucinogenic effects. [11].

\subsection{Species with Calcium Oxalate Crystals}

Upon mechanical stimulation, as occurs with chewing, crystalline calcium oxalate needles, bundled in needle-like raphides, release from their intracellular packaging (idioblasts) in a projectile fashion. These needles penetrate the mucous membranes and induce the release of histamine and other inflammatory mediators. [3]

Philodendron species belongs to the Araceae family and are mostly climbing vines with aerial roots. The adult leaves are often large with variable forms and injurious by its insoluble calcium oxalate crystals. It is probably the most popular indoor houseplant in the United States. Substantial ingestion may cause some gastrointestinal irritation including nausea, vomiting, and diarrhea. Most cases result in no symptoms. [12]

\subsection{Species with Cardiac Glycosides}

Cardioactive steroids, termed cardiac glycosides when 
sugar moieties are attached, inhibit the cellular $\mathrm{Na}^{+} / \mathrm{K}^{+}$-ATPase. The effect is to indirectly increase intracellular $\mathrm{Ca}^{2+}$ concentrations in certain cells, particularly myocardial cells. Therapeutically, this both enhances cardiac inotropy (contractility) and slows the heart rate. However, excessive elevation of the intracellular $\mathrm{Ca}^{2+}$ also increases myocardial excitability, predisposing to the development of ventricular dysrhythmias. Enhanced vagal tone, mediated by the neurotransmitter acetylcholine, is common with poisoning by these agents, and results in bradycardia and heart block. [3]

Digitalis purpurea L. belongs to the family Plantaginaceae and is commonly known as foxglove, thimble herb or troches. It is native to the Madeira archipelago and is also present in mainland Portugal, as well as in Europe. It can be found in sheltered rock outcrops, embankments with grasses, borders, clearings or scrub. [13]

All parts of the plant show toxicity due to the presence of cardiotoxic glycosides, namely digitoxin, gitoxin and gitaloxin. [13]

Its typical symptoms of this intoxication are extreme fatigue, visual disturbances, weakness, nausea, headaches and abdominal pain, strange dreams, among others, however in this case the symptoms will depend on the type of poisoning, if acute the symptoms will be nausea, vomiting and cardiovascular effects including arrhythmias. If intoxication is chronic the typical symptoms will be weakness and changes in vision. [3]

The treatment will depend on the symptoms manifested and the changes in the electrocardiogram, but will be essentially a supportive treatment until the levels of digoxin return to the normality. [14]

Intoxication by $D$. purpurea cases is usually accidental, as this plant can be confused with other edible plants for consumption. There are reported cases of suicide attempts, which can be fatal. [14]

\subsection{Species with Convulsant Poisons and With Nicotine-Like Alkaloids}

A convulsion is the rhythmic, forceful contraction of the muscles, one cause of which is seizures. Seizures are disorganized discharges of the central nervous system that generally, but not always, result in a convulsion. There are various toxicological mechanisms that result in seizures including antagonism of gamma-aminobutyric acid (GABA) at its receptor on the neuronal chloride channel, imbalance of acetylcholine homeostasis, excitatory amino acid mimicry, sodium channel alteration, or hypoglycemia. Strychnine and its analogues antagonize the postsynaptic inhibiting activity of glycine at the spinal cord motor neuron. Strychnine results in hyperexcitability of the motor neurons, which manifests as a convulsion. [3]

Conium maculatum L. belonging to the Apiaceae family is commonly known as hemlock. It is a native species of
Europe and Asia, being also autochthonous in Portugal, mainly in the center and north of the country. It can be found in vacant lots, road berms and water courses on humid substrates and with organic matter. $[4,8]$

Its toxicity is due to the presence of various pyridine alkaloids, and at least ten different types of alkaloids have already been identified. These alkaloids have a characteristic smell, which may allow identifying the plant. Of all alkaloids, the most abundant and also responsible for cases of plant toxicity are conine or cicutoxin and gamma-conicein. The whole plant shows toxicity, however its seeds and fruits are more toxic than the remaining parts of the plant. It should be noted that the dry plant presents less toxicity. [15]

It is known that these alkaloids alter the functions of the central nervous system, since they can bind to the nicotinic acetylcholine receptors. Thus, the symptoms of intoxication include sluggish movement, salivation, mydriasis, tachycardia, rapid pulse, hyperventilation, respiratory and cardiac system depression, coma and death. Treatment is also supportive as there is no antidote. Intubation, gastric decontamination, and anti-convulsants may be given if necessary. [15]

Most of the cases are accidental intoxications; however this plant has been widely used throughout history as poison used to kill for example prisoners. The best known case is that of Socrates in the year 399 BC. [16]

The oral conine ED 50 (median effective dose) capable of inducing mortality in the rat is $100 \mathrm{mg} / \mathrm{kg}$, and the dose of gamma conicein is only $12 \mathrm{mg} / \mathrm{kg}$. It is also known that this plant is more toxic in the spring, due to a higher content of gamma conicein. [16]

Nicotiana glauca Graham belongs to the Solanaceae family is known as the tobacco tree and belongs to the same genus as the Nicotiana tabacum L., the plant from which tobacco originated. Morphologically they can be distinguished by the fact that $N$. glauca has leaves with a smooth surface, without trichomes and presents yellow flowers. [17]

This plant has exotic naturalness, originating in the South of America. It currently exists in the American continent (California, Mexico), Australia, in the Mediterranean and also in some regions of Portugal, having been an introduced species. It can be found on roadsides and roads, in rubble and wasteland. $[8,17]$

The leaves and bark of the plant have a toxic pyridine alkaloid, anabasin, which is a structural analogue of nicotine. [18]

Anabasin has been described as causing severe and sometimes fatal intoxication in humans due to the ingestion of its leaves, since this plant is easily confused with other edible plants, namely spinach (Spinacia oleracea L.). [18]

This compound induces a nicotine-cholinergic syndrome in which the occurrence of vomiting, muscular weakness, loss of consciousness and respiratory failure occurs. [17] 
The onset of symptoms is relatively rapid and may appear 2 hours after ingestion of the leaves. In this case there is also no antidote and the treatment can only be performed through mechanical ventilation until symptoms are absent. [17]

\subsection{Species with Mitotic Inhibitors}

These agents interfere with the polymerization of microtubules, which must polymerize for mitosis to occur, leading to metaphase arrest. Rapidly dividing cells (e.g., gastrointestinal or bone marrow cells) typically are affected earlier and to a greater extent than those cells that divide slowly. In addition, microtubules are important in the maintenance of proper neuronal function. [3]

Catharanthus roseus (L.) G. Don, belonging to the family Apocynaceae, is commonly known as Madagascar periwinkle, vinca (formerly known as Vinca rosea). Its toxicity is due to the presence of Vinca alkaloids (e.g., vincristine), clinically similar to colchicine, a cytotoxic alkaloid capable of inhibiting microtubule formation.

A tea made from the leaves and stems is used in folk medicine. The uncontrolled ingestion may cause initial oropharyngeal pain followed in several hours by intense gastrointestinal symptoms. Vinca alkaloids may subsequently produce peripheral neuropathy, bone marrow suppression, and cardiovascular collapse. [19]

Colchicum species are members of the Liliaceae family and are cultivated for their long, tubular, purple or white flowers, which emerge from the underground bulb. Colchicum autumnale $\mathrm{L}$. is a species that produces flowers in the following autumn, which then die back, and its leaves appear only during the spring. Colchicum species are grown outdoors in gardens. C. autumnale is a commercial source for medicinal colchicine. Its toxicity is due to the presence of colchicine, a cytotoxic alkaloid capable of inhibiting microtubule formation. The ingestion may cause initial oropharyngeal pain followed after several hours by intense gastrointestinal symptoms. Colchicine may subsequently produce peripheral neuropathy, bone marrow suppression, and cardiovascular collapse. [20]

\subsection{Species with Pyrrolizidine Alkaloids}

Pyrrolizidine alkaloids are metabolized to pyrroles, which are alkylating agents that injure the endothelium of the hepatic sinusoids or pulmonary vasculature. Endothelial repair and hypertrophy result in veno-occlusive disease. Centrilobular necrosis may occur following acute, high-dose exposures, presumably caused by the overwhelming production of the pyrrole. Chronic use is also associated with hepatic carcinoma. [3]

Senecio vulgaris L., belonging to the family Asteraceae, is commonly known as groundsel, annual grows to about 1 foot high. The leaves are soft, fleshy, and somewhat lobed. The flowers are golden-yellow. It is a European plant that has become naturalized as a weed in Alaska, and most of the continental United States. Its toxicity is due to the presence of pyrrolizidine alkaloids. Substantial short-term exposure may cause acute hepatitis, and chronic exposure to lower levels (including Senecio in herbal teas) may cause hepatic veno-occlusive disease and in some cases pulmonary hypertension. [21]

\subsection{Species with Sodium Channel Activators}

These agents stabilize the open form of the voltage-dependent sodium channel in excitable membranes, such as neurons and the cardiac conducting system. This causes persistent sodium influx (i.e., persistent depolarization) and prevents adequate repolarization leading to seizures and dysrhythmias, respectively. In the heart, the excess sodium influx activates calcium exchange, and the intracellular hypercalcemia increases both inotropy and the potential for dysrhythmias. [3]

Aconitum spp., which are responsible for numerous cases of poisoning, some of them lethal, belonging to the family Ranunculaceae. Most cases occur in eastern countries, namely China and Japan; however the species Aconitum nappellus L. exists in Portugal, but no cases of intoxication in humans are reported. [22]

Aconitine and related alkaloids, sodium channel activators, are responsible for the toxicity. The symptoms are predominantly neurological and cardiac. There is transient burning in the mouth after ingestion, followed after several hours by increased salivation, vomiting and diarrhea. The patient may complain of headache, muscular weakness, and dimness of vision. Bradycardia and other cardiac dysrhythmias can be associated with severe blood pressure abnormalities. Coma may develop, and convulsions may be a terminal event. [23]

\subsection{Species with Cyanogenic Compounds}

Cyanogenic compounds, most commonly glycosides, must be metabolized to release cyanide. Cyanide inhibits the final step of the mitochondrial electron transport chain, resulting rapidly in cellular energy failure. [3]

Hydrangea macrophylla (Thunb.) Ser. is a species of the family Hydrangeaceae native to Japan. The common names include bigleaf hydrangea, lacecap hydrangea, and hortensia. It is widely cultivated in many parts of the world in many climates. The flower bud is poisonous due to the existence of hydrangin, a cyanogenic glycoside.

Because the cyanogenic glycosides must be hydrolyzed in the gastrointestinal tract before cyanide ion is released, several hours may elapse before poisoning occurs. Abdominal pain, vomiting, lethargy, and sweating typically should occur first. In severe poisonings, coma may develop and may be accompanied by convulsions and cardiovascular collapse. [3]

\subsection{Species with Toxalbumins}

The protein toxins derived from these plants work 
specifically by inhibiting the function of ribosomes, the subcellular organelle responsible for protein synthesis. The toxins typically have two linked polypeptide chains. One of the chains binds to cell-surface glycoproteins to allow endocytosis into the cell. The other chain upon cell entry binds the $60 \mathrm{~S}$ ribosomal subunit and impairs its ability to synthesize protein. [3]

Ricinus communis L., belonging to the family Euphorbiaceae, is commonly known as castor, castor bean, among others. It is also a plant of exotic origin, probably originating in Africa and can be found in Portugal, mainly in coastal regions. It is an infesting plant, which was introduced and that develops in vacant lots, agricultural fields, banks and beds of water lines. The seeds are the part of the plant that shows toxicity. Its toxicity is due to the presence of a protein called ricin. Ricin is a ribosome inactivating protein, being considered a cytotoxic protein, due to the fact that it prevents the protein translation. [3]

In the case of this plant there are several ways of exposure, with different possible symptoms. The ways of exposure may be ingestion, namely seeds, inhalation, since from the seeds it is possible to do the purification of ricin and obtain a white powder that can easily be inhaled and injected. In less severe cases of intoxication by ingestion, only nausea, emesis, diarrhea and abdominal pain may occur, however, in more severe cases dehydration, hypovolemic shock, renal and hepatic failure may occur and in severe cases death may occur 36 to 72 hours after ingestion of the seeds. [3]

Inhalation and injection are considered the most lethal ways of exposure. In the case of inhalation, symptoms may manifest after 8 hours and include coughing, fever, and there may be progression to respiratory failure and death. In the case of injection, the symptoms have an onset six or less hours after exposure and are manifested by generalized weakness, myalgias, vomiting hypotension and by generalized organ failure and consequent death. [24]

Some dermatological toxicity can be observed due to the plant's contact with the skin, namely urticaria. Treatment of this type of poisoning is also a supportive treatment as there is no antidote. Treatment may include administration of vasoconstrictors, such as dopamine, activated charcoal, and gastric lavage. [24]

Despite this, studies have been carried out in the field of neutralizing the ricin toxin, for which, as previously mentioned, there is no antidote. Ricin is easily purifiable and in large quantities presents a great risk to public health. There are currently two vaccines under development (Phase I clinical trials) that are based on the ricin RTA subunit: RiVax and RVEc. [25, 26]

Through previous cases of intoxication from ricin intake, it is known that the lethal dose estimated in humans should be from 1 to $20 \mathrm{mg} / \mathrm{kg}$, i.e., approximately 8 seeds. The number of seeds will vary according to their size, content, time of year, among others. [24]

There are cases of accidental intoxication by ingestion of ricin seeds mainly by children and animals and also cases of intentional poisoning usually associated with suicide attempts and bioterrorism. In 2003, ricin was found in the United States in a letter to the White House. $[27,28]$

Abrus precatorius L., belonging to the family Fabaceae, with the common names of bead vine, black eyed Susan, coral bead. Its toxicity is due to the presence of abrin. Abrin is a toxic protein present in the seeds of these plants and is considered one of the most lethal poisons. The toxin, a plant lectin (toxalbumin), inhibits cellular protein synthesis, and is contained within the hard, water-impermeable coat of the seeds. The toxin is not released unless the seed is chewed and digested or the seed coat is otherwise broken. [3]

It is estimated that the fatal dose for the human being is between 0.1 and $1 \mu \mathrm{g} / \mathrm{kg}$ abrin. [29]

The typical symptoms of this intoxication are at the gastro intestinal level, i.e., nausea, vomiting, abdominal pain and diarrhea. [30]

Most cases are accidental, especially in children who tend to ingest the berries of this plant. There are also cases of attempted suicide with this plant. $[29,30]$

\section{Conclusions}

Toxic plant poisoning can be considered a public health problem. Throughout this work, we can verify that intoxication by toxic plants are a significant cause of morbidity and mortality, which in most cases are accidental intoxications, therefore may be avoidable. We have also verified that knowledge is the best form of prevention and therefore one of the greatest challenges for the future will be to educate and raise awareness among the general population about this problem, as well as informing and reporting these cases of intoxication so that health professionals have more sensitivity and willingness to treat them.

The knowledge about toxic plants is still insufficient and can always use additional efforts to both: deepen research and understanding of these properties and how they can be countered with antidotes or used as part of herbal medicines in the right proportions; and to spread word and awareness about the properties of these plants among populations.

\section{Acknowledgements}

This work was funded, in part, by iMed.ULisboa (UID/DTP/04138/2013) from Fundação para a Ciência e a Tecnologia (FCT), Portugal.

\section{REFERENCES}

[1] M. C. Mendieta, A. D. Z. De Souza, S. Ceolin, N. R. C. 
Vargas, T. Ceolin, R. M. Heck. Toxic plants: importance of knowledge for realization of health education, Journal of Nursing UFPE On Line, Vol.8, Nº3, 680-686, 2014.

[2] Sistema Nacional de Informações Tóxico-Farmacológicas (Sinitox), Online available from http://dados.contraosagrotoxicos.org/pt PT/dataset/intoxic acoes-sinitox

[3] L. S. Nelson, R. D. Shih, M. J. Balick. Handbook of Poisonous and Injurious Plants. Second Edition, New York Botanical Garden Press/Springer-Verlag, New York, US, 2007.

[4] Flora de Portugal interactiva, Online available from http://www.flora-on.pt

[5] M. A. Berdai, S. Labib, K. Chetouani, M. Harandou. Atropa belladonna intoxication: A case report, Pan African Medical Journal, Vol.11, 72, 2012.

[6] P. Joshi, A. C. Wicks, S. K. Munshi. Recurrent autumnal psychosis, Postgraduate Medical Journal, Vol.79, N 930 , 239-40, 2003.

[7] S. T. Martinez, M. R. Almeida, A. C. Pinto. Natural hallucinogens: a flight from Medieval Europe to Brazil, Química Nova, Vol.32, Nº, 2501-2507, 2009.

[8] T. A. López, M. S. Cid, M. L. Bianchini. Biochemistry of hemlock (Conium maculatum L.) alkaloids and their acute and chronic toxicity in livestock. A review, Toxicon, Vol.37, $\mathrm{N}^{\mathrm{o}} 6,841-65,1999$.

[9] S. A. Adegoke, L. A. Alo. Datura stramonium poisoning in children, Nigerian Journal of Clinical Practice, Vol.16, $\mathrm{N}^{\circ} 1$, 116-8, 2013.

[10] B. Şanlıdağ, O. Derinöz, N. Yıldız. A case of pediatric age anticholinergic intoxication due to accidental Datura stramonium ingestion admitting with visual hallucination, The Turkish Journal of Pediatrics, Vol.56, №3, 313-315, 2014.

[11] A. Alizadeh, M. Moshiri, J. Alizadeh, M. Balali-Mood. Black henbane and its toxicity - a descriptive review. Avicenna Journal of Phytomedicine, Vol.4, Nº5, 297-311, 2014.

[12] R. Mrvos, B. S. Dean, E. P. Krenzelok. Philodendron / Dieffenbachia ingestions: Are they a problem? Journal of Toxicoly. Clinical Toxicology, Vol.29, 485-491, 1991.

[13] E. Lacassie, P. Marquet, S. Martin-Dupont, J.M. Gaulier, G. Lachâtre. A non-fatal case of intoxication with foxglove, documented by means of liquid chromatography electrospray - mass spectrometry, Journal of Forensic Sciences, Vol. 45, №5, 1154-1158, 2000.

[14] A. Joost. Intoxication by Digitalis purpurea in suicidal intention - a case report. Revista Societatii de Medicina Interna, $\mathrm{N}^{\mathrm{o}} 4$ din luna, 2010.

[15] B. S. Frank, W. B. Michelson, K. E. Panter, D. R. Gardner. Ingestion of poison hemlock (Conium maculatum), Western Journal of Medicine, Vol.163, Nº6, 573-574, 1995.
[16] T. Reynolds. Hemlock alkaloids from Socrates to poison aloes, Phytochemistry, Vol.66, Nº12, 1399-1406, 2005.

[17] D. Ntelios, M. Kargakis, T. Topalis, A. Drouzas, E. Potolidis. Acute respiratory failure due to Nicotiana glauca ingestion, Hippokratia, Vol.17, No2, 183-184, 2013.

[18] V. Furer, M. Hersch, N. Silvetzki, G. S. Breuer, S. Zevin Nicotiana glauca (Tree Tobacco) Intoxication-Two Cases in One Family, Journal of Medical Toxicology, Vol.7, Nº1, 47-51, 2011.

[19] M. L. Wu, J. F. Deng, J. C. Wu, F. S. Fan, C. F. Yang. Severe bone marrow depression induced by an anticancer herb Catharanthus roseus, Journal of Toxicology. Clinical Toxicology, Vol.42, 667-671, 2004.

[20] V. C. Danel, J. F. Wiart, G. A. Hardy, F. H. Vincent, N. M. Houdret. Self-poisoning with Colchicum autumnale L. flowers, Journal of Toxicology. Clinical Toxicology, Vol.39, 409-411, 2001.

[21] A. Ortiz Cansado, E. Crespo Valadés, P. Morales Blanco P, J. Sáde Santamaria, J. M. González Campillejo, T. Ruiz Téllez. Veno-occlusive liver disease due to intake of Senecio vulgaris tea, Gastroenterology and Hepatology, Vol.18, 413-416, 1995.

[22] K. Kazuma, M. Satake, K. Konno. Case of fatal aconite poisoning, and its background, Shokuhin Eiseigaku Zasshi. Journal of the Food Hygienic Society of Japan, Vol.54, No 6 , 419-425, 2013.

[23] C. C. Lin, T. Y. Chan, J. F. Deng. Clinical features and management of herb-induced aconitine poisoning, Annals of Emergency Medicine, Vol.43, 574-579, 2004.

[24] J. Audi, M. Belson, M. Patel, J. Schier, J. Osterloh J. Ricin Poisoning: A comprehensive review, JAMA, Vol.9, 2342-2351, 2005.

[25] K. Jasheway, J. Pruet, E. V. Anslyn, J. D. Robertus Structure-Based Design of Ricin Inhibitors, Toxins (Basel), Vol.3, No $10,1233-1248,2011$.

[26] J. M. O’Hara, R. N. Brey, N. J. Mantis. Comparative efficacy of two leading candidate ricin toxin A subunit vaccines in mice, Clinical and Vaccine Immunology, Vol.20, Nº, 789-794, 2013.

[27] S. Worbs, K. Köhler, D. Pauly, M. A. Avondet, M. Schaer, M. B. Dorner, B. G. Dorner. Ricinus communis intoxications in human and veterinary medicine-a summary of real cases, Toxins (Basel), Vol.3, No 10, 1332-1372, 2011.

[28] D. R. Cardoso, T. A. O. Cardoso. Bioterrorism: data of a recente history of risks and uncertainties, Ciência \& Saúde Coletiva, Vol.16, 821-830, 2011.

[29] M. Alhamdani, B. Brown, P. Narula. Abrin Poisoning in an 18-Month-Old Child, American Journal of Case Reports Vol.16, 146-148, 2015.

[30] D. H. Jang, R. S. Hoffman, L. S. Nelson. Attempted Suicide, by Mail Order: Abrus precatorius, Journal of Medical Toxicology, Vol.6, Nº4, 427-430, 2010. 